${ }^{1}$ Fonoaudióloga. Magíster en Salud Pública, Universidad de Chile.

Clínica Alemana de Santiago, Universidad del Desarrollo. Santiago. Chile.

${ }^{2}$ Neurólogo. Unidad de Neurología Vascular, Departamento de Neurología y Psiquiatría Clínica Alemana de Santiago,Universidad del Desarrollo. Departamento de

Ciencias Neurológicas, Facultad de Medicina, Universidad de Chile. Santiago. Chile. ${ }^{3}$ Neurólogo. Unidad de Neurología Vascular, Departamento de Neurología y Psiquiatría Clínica Alemana de Santiago, Universidad del Desarrollo. Santiago. Chile.

Fuente de apoyo financiero: no

Recibido el 14 de julio de 2016, aceptado el 31 de enero de

Correspondencia a: Francisca González Mc. franciscagonzalezmc@gmail.com

\section{Incidencia poblacional, características epidemiológicas y desenlace funcional de pacientes con ataque cerebrovascular isquémico y afasia}

\author{
FRANCISCA GONZÁLEZ MC. ${ }^{1}$, PABLO LAVADOS G. ${ }^{2}$, \\ VERÓNICA OLAVARRÍA I. ${ }^{3}$
}

\section{Incidence of aphasia in patients experiencing an ischemic stroke}

Background: Sequelae after a stroke are common and may lead to disability. Aphasia - defined as an acquired language disturbance - can cause important limitations in quality of life. Aim: To describe the epidemiological features of patients who had an aphasia after a first episode of ischemic stroke and their functional outcome at six months. Material and Methods: Review of a database of a population study on the incidence, 30-day case fatality rate, and prognosis of stroke performed in a northern Chilean city between 2000 and 2002. Results: Aphasia was diagnosed in 28 of 142 patients in whom the disorder was sought (20\%). The projected incidence rate in the city where the study was carried out is 7.06 per 100,000 inhabitants. The mean age of these 28 patients was $66 \pm 20$ years and $53 \%$ were women. The main risk factor for stroke was hypertension in $62 \%$. The etiology of stroke was undetermined in $64 \%$ of these patients. Partial anterior circulation infarction was the most common stroke location in $61 \%$. Conclusions: Twenty percent of patients with a first episode of ischemic stroke have aphasia.

(Rev Med Chile 2017; 145: 194-200)

Key words: Aphasia; Epidemiology; Incidence; Stroke. hile ha experimentado cambios poblacionales importantes en los últimos años que han generado significativas modificaciones en la estructura de la población. Las enfermedades no transmisibles son las responsables de las principales causas de morbi-mortalidady se relacionan con el envejecimiento de nuestra población, cuya esperanza de vida promedio alcanza los 79 años ${ }^{3}$. El ataque cerebrovascular (ACV) constituye un ejemplo de lo anterior. Desde hace algunos años es la primera causa de muerte en nuestro país, representando el $9 \%$ de las muertes en el año $2009^{4}$.
Tomando en consideración estos cambios en la estructura de la población chilena, durante los años 2000 y 2002 se realizó en la ciudad de Iquique un estudio poblacional, epidemiológico prospectivo sobre ACV, que aportó información relevante al país. Este trabajo de investigación, PISCIS (Proyecto Investigación de Stroke en Chile: Iquique Stroke Study) Incidence, 30 -day case-fatality rate, and prognosis of stroke in Iquique: a 2-year community-based prospective study, encontró, una tasa promedio ajustada por edad de primer evento cerebrovascular de 140,1 por 100.000 habitantes. Se identificó que $18 \%$ presentó una discapacidad 
de grado moderado o severo luego de los 6 primeros meses de evolución ${ }^{2}$.

Desde esta perspectiva, esta enfermedad es un generador de importantes y diversas secuelas tanto del ámbito motor como cognitivo, que interfieren significativamente la calidad de vida de quienes la presentan. Una de estas secuelas es la afasia, trastorno adquirido de la comunicación que se observa frecuentemente en la práctica diaria. Según datos nacionales entregados por el Ministerio de Salud, la incidencia de afasia post ACV en etapa aguda, varía entre $20 \%$ a $38 \%{ }^{4}$. Si bien en Chile, se han realizado esfuerzos en los últimos años para conocer más profundamente esta patología, las características de los pacientes, subtipos y evolución de los enfermos a través del tiempo, la información disponible aún es limitada. Es por esta razón que nuestro objetivo principal fue determinar la incidencia de la afasia en esa población, describir sus características epidemiológicas y conocer el desenlace funcional de estos pacientes a los seis meses de seguimiento, medido con la escala de Rankin modificada.

\section{Material y Método}

Esta es una investigación de tipo observacional, descriptivo, de alcance transversal y enfoque cuantitativo. Se revisó en forma retrospectiva la base de datos del estudio poblacional PISCIS el cual fue realizado en la ciudad de Iquique, Chile, entre los años 2000 y $2002^{2}$. La población en estudio estuvo compuesta por todos los pacientes con diagnóstico de un primer episodio de ACV isquémico ocurrido durante los años 2000-2002, que ingresaron a este estudio poblacional. Se definió ACV como "déficit neurológico focal o global, de inicio súbito, no convulsivo, que dura más de 24 horas y que se debe a una alteración vascular"5. Una descripción detallada de la metodología del estudio PISCIS en el subgrupo de infartos ha sido reportada previamente ${ }^{2}$.

Variables e instrumentos: Se revisó la base de datos de este estudio -previamente aprobado por el Comité de ética respectivo- con la información que ésta contiene y no se accedió al registro de las fichas clínicas de los pacientes participantes. Esta información fue organizada en forma anonimizada para proteger y resguardar las identidades de los pacientes.
Se seleccionaron y analizaron las variables edad, sexo y los siguientes factores de riesgo cardiovascular: hipertensión arterial (HTA), diabetes mellitus (DM), cardiopatía coronaria (CC), insuficiencia cardiaca congestiva (ICC), fibrilación auricular (FA), dislipidemia, consumo de tabaco y alcohol. Se identificó la etiología delACV mediante los criterios TOAST en: cardioembólico, aterotrombótico, lacunar, no determinada y otra ${ }^{6}$. La tipología del ACV se clasificó de acuerdo a los criterios clínicos de Bamford en: circulación anterior total (TACI); circulación anterior parcial (PACI), circulación posterior (POCI) y lacunar (LACI $)^{7}$.

La escala de Rankin modificada ${ }^{8}$ fue aplicada a los pacientes del estudio a 6 meses de seguimiento, con el fin de determinar el impacto funcional y la recuperación tras el ACV. Se seleccionó esta prueba por estar validada para la aplicación en el $\mathrm{ACV}$, ser de fácil reproducción y de amplio uso en estudios neurológicos, especialmente en ensayos clínicos (Tabla 1). Esta escala ordinal posee un puntaje que va de 0 a 6 , donde el puntaje 0 corresponde a aquel paciente que no presenta secuela alguna y el puntaje 6 indica muerte.

Se calcularon las tasas de incidencia de la afasia en distintos grupos de edad y la incidencia total para la ciudad de Iquique, considerando como

Tabla 1. Escala de Rankin Modificada

\begin{tabular}{|cl|}
\hline Puntaje & \\
0 & Sin síntomas \\
1 & $\begin{array}{l}\text { Sin dishabilidad significativa a pesar de los } \\
\text { síntomas; el paciente es capaz de llevar a cabo } \\
\text { las actividades }\end{array}$ \\
2 & $\begin{array}{l}\text { Dishabilidad leve; el paciente es incapaz de lle- } \\
\text { var a cabo todas las habilidades previas, pero } \\
\text { es capaz de cuidar de sus asuntos sin ayda }\end{array}$ \\
3 & $\begin{array}{l}\text { Dishabilidad moderada; el paciente requiere } \\
\text { de alguna ayuda, pero es capaz de caminar } \\
\text { sin asistencia }\end{array}$ \\
4 & $\begin{array}{l}\text { Dishabilidad moderada - severa; incapaz de } \\
\text { caminar sin ayuda y requerimiento de asis- } \\
\text { tencia }\end{array}$ \\
5 & $\begin{array}{l}\text { Dishabilidad severa; paciente postrado en } \\
\text { cama que requiere cuidados de enfermería y } \\
\text { atención constante }\end{array}$ \\
\hline 6 & \begin{tabular}{l} 
Paciente muerto \\
\hline
\end{tabular}
\end{tabular}

Banks J, Charles A, Marotta A. 2007. 
denominador dos poblaciones: la población proyectada para el año 2000 de acuerdo a los resultados del Censo año 1992 y la población de Iquique según resultados del Censo correspondiente al año 2002 (utilizadas en el estudio PISCIS). Se han estandarizado las tasas a la población mundial, mediante el método directo.

\section{Análisis de resultados}

Para realizar la exploración de la información, se analizaron descriptivamente los datos por variable, usando frecuencias, porcentajes, promedios y desviación estándar. Se generaron tablas de contingencia, aplicando la prueba de $\chi^{2}$.

Para la comparación de grupos, se utilizó la prueba de $\chi^{2}$ con un nivel de significancia de alfa menor de 0,05 y se calculó t-student. Todos los análisis fueron realizados utilizando el programa computacional SPSS, versión 20.

\section{Resultados}

\section{Afasia en pacientes con infarto cerebral}

Del total de pacientes con infarto cerebral $(\mathrm{n}=182), 142$ fueron clasificados en afásicos o no afásicos; de estos, 28 pacientes fueron diagnosticados con afasia $(19,7 \%)$.

La tasa de incidencia total de afasia fue de 7,06 por 100.000 habitantes y la mayor incidencia de afasia se encontró en el grupo de 75 a 84 años de edad, con una tasa de 125,67 por 100.000 habitantes (Tabla 2).

\section{Etiología del ACV isquémico en pacientes con y sin afasia}

La Figura 1 muestra la distribución según etiología del ACV isquémico y la presencia o ausencia de afasia. La etiología indeterminada fue la causa más frecuente de infarto tanto en los pacientes con y sin diagnóstico de afasia, observando $64,3 \%$ y $48,2 \%$, respectivamente $(\mathrm{p}=0,12)$. En lo que respecta los pacientes afásicos, la etiología de tipo cardioembólica representó la segunda causa con $28,6 \%$ y la etiología de pequeño vaso sólo $7,1 \%$.

\section{Tipo de ACV y presencia de afasia}

Respecto al tipo clínico de ACV, la Figura 2 muestra las frecuencias en ambos grupos de pacientes. Se observó que el 60,7\%, de los pacientes con afasia, tenía ACV de circulación anterior
Tabla 2. Tasas de incidencia por 100.000 habitantes en pacientes afásicos, según grupos de edad

\begin{tabular}{|lcc|}
\hline $\begin{array}{l}\text { Grupos de edad } \\
\text { (años) }\end{array}$ & $\begin{array}{c}\text { n casos/ } \\
\text { n en riesgo }\end{array}$ & $\begin{array}{c}\text { Tasa de } \\
\text { incidencia }\end{array}$ \\
\hline $0-24$ & $1 / 179.054$ & 0,56 \\
$25-34$ & $1 / 66.462$ & 1,5 \\
\hline $35-44$ & $2 / 62.300$ & 3,21 \\
$45-54$ & $2 / 43.174$ & 4,63 \\
\hline $55-64$ & $6 / 23.682$ & 25,33 \\
$65-74$ & $6 / 13.838$ & 43,36 \\
$75-84$ & $8 / 6.366$ & 125,67 \\
$\geq 85$ & $2 / 1.836$ & 108,93 \\
\hline Total & $28 / 396.712$ & 7,06 \\
\hline $\begin{array}{l}\text { Ajustada a Chile } \\
\text { (año 2002) }\end{array}$ & & 9,5 \\
\hline $\begin{array}{l}\text { Ajustada a población } \\
\text { mundial OMS }\end{array}$ & & 8,8 \\
\hline
\end{tabular}

Fuente: Elaboración propia, con base en la población total de acuerdo al Censo 2002.

parcial, seguido de infarto de circulación anterior total en $35,7 \%$. Por el contrario, en el grupo sin afasia predomina el infarto lacunar $(46,3 \%)$.

\section{Características demográficas y factores de riesgo} cardiovascular en pacientes afásicos y no afásicos

La edad promedio de los pacientes afásicos fue de 65,8 años (SD 19,7) y la de los no afásicos fue de 65,6 años (SD 14,6). Se observó una tasa de incidencia de la afasia de 7,06 por 100.000 habitantes $y$ la mayor incidencia se encontró en el grupo entre 75 y 85 años con una tasa de 125,67 por 100.000 habitantes. En el grupo de 85 años y más, la tasa fue de 108,93 por 100.000 habitantes. En relación al género, el $46,4 \%$ de los pacientes con afasia fue de sexo masculino y el $53,6 \%$ de sexo femenino $(\mathrm{p}=0,35)$. En aquellos sin afasia, predominó el sexo masculino con $56,1 \%$ (Tabla 3 ).

\section{Desenlace funcional en pacientes afásicos y no afásicos}

A los 6 meses, el 33\% de los pacientes afásicos y el $59,8 \%$ de los no afásicos presentaron puntaje entre 0 y 2 en la mRS ( $p=0,01)$; es decir, se encontraban sin mayores secuelas y fueron considerados autovalentes e independientes en sus actividades 

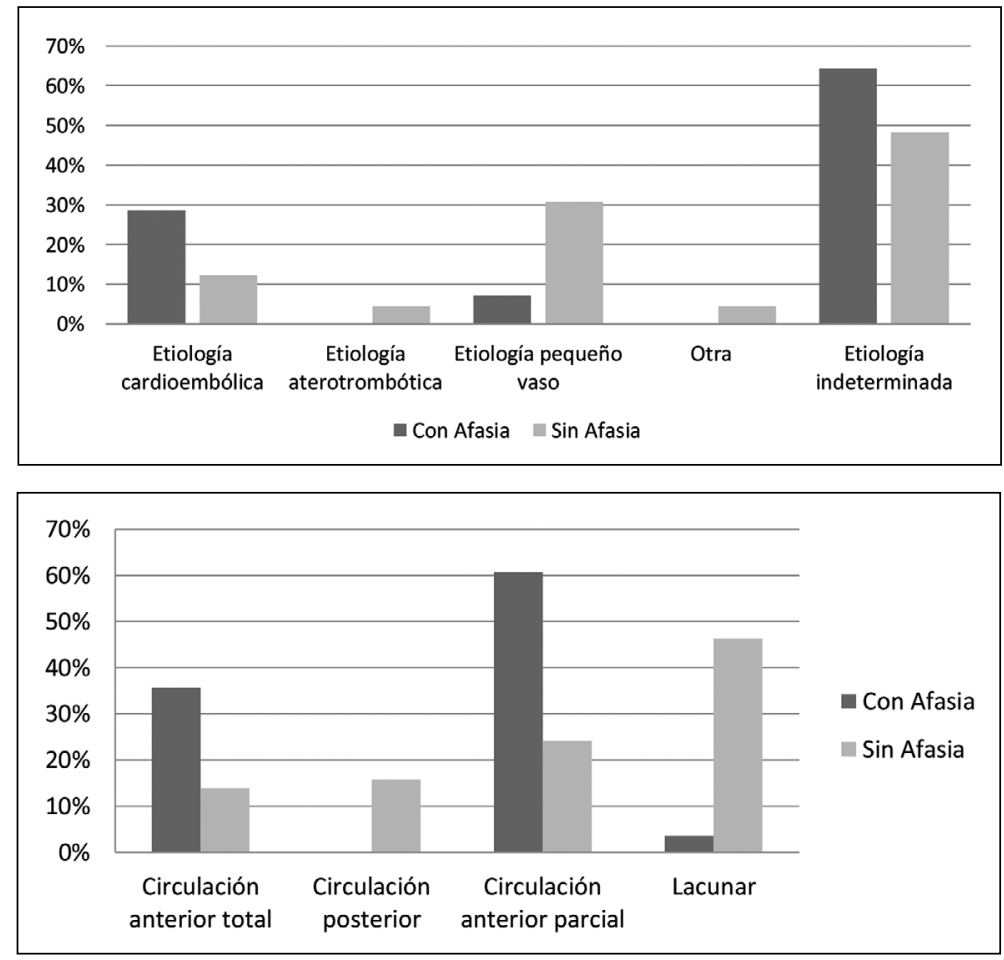

Figura 1. Distribución según etiología de ACV isquémico y presencia/ausencia de afasia.

Tabla 3. Características demográficas y factores de riesgo cardiovascular en pacientes con y sin afasia

\begin{tabular}{|c|c|c|c|c|c|}
\hline \multirow[t]{2}{*}{ Variables } & \multicolumn{2}{|c|}{ Con Afasia } & \multicolumn{2}{|c|}{ Sin Afasia } & \multirow[t]{2}{*}{$\mathbf{p}$} \\
\hline & $\mathbf{n}$ & (\%) & $\mathbf{n}$ & (\%) & \\
\hline \multicolumn{6}{|l|}{ Sexo } \\
\hline Masculino & 13 & 43 & 64 & 56 & 0,35 \\
\hline Edad (años) & & & & & 0,52 \\
\hline $0-24$ & 1 & $(3,6)$ & 1 & $(1,5)$ & \\
\hline $25-34$ & 1 & $(3,6)$ & 1 & $(0,9)$ & \\
\hline $35-44$ & 2 & $(7,14)$ & 5 & $(4,4)$ & \\
\hline $45-54$ & 2 & $(7,14)$ & 18 & $(15,8)$ & \\
\hline $55-64$ & 6 & $(21,4)$ & 29 & $(25,4)$ & \\
\hline $65-74$ & 6 & $(21,4)$ & 30 & $(26,3)$ & \\
\hline $75-84$ & 8 & $(28,57)$ & 24 & $(21,1)$ & \\
\hline$\geq 85$ & 2 & $(7,14)$ & 5 & $(4,4)$ & \\
\hline \multicolumn{6}{|c|}{ Factores de riesgo } \\
\hline HTA & 16 & $(61,5)$ & 74 & $(67,3)$ & 0,57 \\
\hline DM & 6 & $(23,1)$ & 29 & $(26,4)$ & 0,73 \\
\hline CC & 1 & $(3,8)$ & 11 & (10) & 0,31 \\
\hline ICC & 1 & $(3,8)$ & 6 & $(5,5)$ & 0,73 \\
\hline FA & 3 & $(11,5)$ & 12 & $(11,2)$ & 0,96 \\
\hline Dislipidemia & 2 & $(8,3)$ & 23 & $(21,9)$ & 0,12 \\
\hline Tabaco & 5 & $(19,2)$ & 18 & $(16,4)$ & 0,7 \\
\hline Alcohol & 4 & $(15,4)$ & 12 & $(10,9)$ & 0,52 \\
\hline
\end{tabular}

HTA: Hipertensión arterial; DM: Diabetes Mellitus tipo 2; CC: Cardiopatía coronaria; IC: Insuficiencia cardiaca; FA: Fibrilación auricular. Fuente: Elaboración propia con base en estudio PISCIS. Iquique, 2000-2002. 


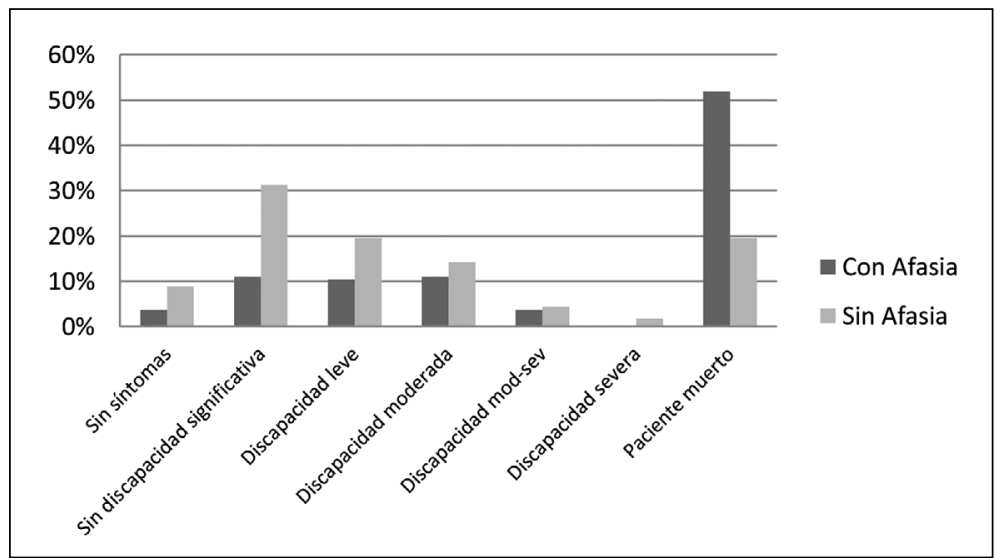

Figura 3. Distribución de porcentajes en pronóstico a los 6 meses post $1 \mathrm{erACV}$ isquémico en pacientes con y sin diagnóstico de afasia. cotidianas ( sin síntomas, sin discapacidad significativa o sólo discapacidad leve) (Figura 3). Un $14,8 \%$ de los pacientes afásicos presentó entre 3 a 5 puntos en la escala de Rankin modificada. Lo anterior indica que presentaban dificultades evidentes al momento de la evaluación, como por ejemplo la necesidad de alguna ayuda técnica o la dependencia total de un tercero.

\section{Discusión}

Este estudio muestra que a nivel poblacional un 19,7\% (IC 95\% 14-27)de los pacientes con infarto cerebral fue diagnosticado con un trastorno de lenguaje adquirido (afasia). Este resultado es algo más bajo a los encontrados en la literatura internacional, en la que se reporta entre $26 \%$ y $30 \%{ }^{9-12}$ pero bastante similar a los reportes mencionados por el Ministerio de Salud de Chile MINSAL1, entre $20 \%$ y $38 \%{ }^{9-12}$ y también a los reportes mencionados por el Ministerio de Salud de Chile MINSAL $^{1}$, entre $20 \%$ y $38 \%$. En el estudio de Croquelois y Bogousslavsky ${ }^{9}$ se analizaron a 1.500 pacientes afásicos en forma consecutiva y se observó que la incidencia de la afasia post ACV isquémico podía presentar variación en su porcentaje y cuyas diferencias fueron estimadas por los mismos autores entre el $14 \%$ y el $38 \%$. Estas dependían principalmente del momento en que se realizaba la evaluación de la comunicación. La evaluación de la afasia de los pacientes del estudio PISCIS fue realizada por el equipo de neurólogos a cargo durante las primeras horas post ataque cerebrovascular, mediante la aplicación de pauta de evaluación estructurada. Hubo un grupo de pacientes (43) a los que fue imposible determinar la presencia del trastorno afásico, ya sea por presentar compromiso importante de conciencia o haber fallecido inmediatamente luego del ACV.

La tasa de incidencia total de afasia en la población estudiada fue mayor en grupos de edad avanzada, lo que concuerda con lo descrito en la literatura, en la que se reporta que la afasia es una patología que aumenta considerablemente con la edad $^{9,12}$.

En relación a las características sociodemográficas de los pacientes estudiados, se encontró que la edad promedio de los pacientes afásicos y no afásicos fue similar. Lo anterior difiere a lo reportado en otros estudios, en los que los pacientes afásicos tienden a ser mayores en edad en comparación a los no afásicos ${ }^{9,14}$. Sin embargo, al revisar la mediana de nuestros datos, ésta es de 70, 5 en los afásicos y 65, 5 en no afásicos, por lo que hay pacientes de más edad entre los afásicos que los no afásicos, lo que concordaría con lo publicado. Con respecto al sexo, la mayoría de los afásicos fue de sexo femenino, similar a lo encontrado en algunos estudios $^{15,16}$, pero difiere de otros en los que se observa un predominio del sexo masculino ${ }^{17}$.

$\mathrm{Al}$ analizar la etiología del ACV isquémico de acuerdo a la clasificación $\mathrm{TOAST}^{6}$, en nuestro estudio se observó que, tanto en los pacientes afásicos como no afásicos, la etiología indeterminada fue la más prevalente. Si bien esta etiología es bastante frecuente en los infartos cerebrales, el porcentaje encontrado en este estudio fue superior a lo expuesto en la literatura. Es muy posible 
que esto se deba a baja tasa de pacientes con un estudio etiológico completo, como lo es por ejemplo la evaluación vascular mediante doppler carotídeo/vertebral y exámenes vasculares de los vasos intra y extracraneales (angiografía por Tomografía Computada, angiografía por Resonancia Magnética o angiografía convencional), así como ecocardiograma, Holter de ritmo cardíaco en busca de fibrilación auricular paroxística, entre otros. Cabe mencionar que si bien han pasado varios años desde la realización del estudio PISCIS, aún existen hospitales públicos a nivel central y en regiones que no cuentan con la tecnología más avanzada para el estudio completo para estos pacientes ni con el recurso humano que se requiere. Probablemente, si esta misma investigación se hiciera actualmente, los resultados en relación a la etiología serían semejantes.

En los pacientes afásicos, la segunda etiología más frecuente correspondió a cardioembólica, observando un porcentaje cercano a $1 / 3$ de los casos. Los estudios publicados sobre este tema han podido concluir que la gran mayoría de estos pacientes presenta esta etiología $a^{9,14,15}$.

De acuerdo al tipo clínico del ACV isquémico, más de $90 \%$ de los pacientes con afasia presentó un ACV de circulación anterior parcial o total. Este resultado es bastante esperable, tomando en consideración que el lóbulo frontal es relevante para la función lingüística, principalmente en lo expresivo, ya que aquí se encuentra el área de Broca, que tiene gran función en lo relacionado al lenguaje.

En relación a la frecuencia de factores de riesgo cardiovascular, los resultados muestran a la hipertensión arterial (HTA) como el gran factor de riesgo presente en los pacientes afásicos, observando un porcentaje de $61,5 \%$, lo que concuerda con lo publicado en la literatura. La Diabetes Mellitus (DM) fue el segundo factor de riesgo encontrado, observando que el 23,1\% de los afásicos la presentaba al momento de su ACV.

Los resultados de la aplicación de la escala de Rankin modificada - instrumento ampliamente utilizado para valorar el desenlace funcional- a los pacientes del estudio PISCIS a los 6 meses de seguimiento, mostraron que el 51,9\% de los pacientes con afasia estaba muerto para la evaluación en ese período. Este resultado concuerda con lo expuesto en la literatura, en la que se señala que la presencia de afasia se asocia a un aumento de la mortalidad y disminución de los rangos de recuperación funcional ${ }^{15,18,19}$. Es posible -entonces- advertirque presentar afasia como secuela post ACV implicaría un peor pronóstico funcional para los pacientes, lo que sería muy interesante de estudiar en mayor profundidad en próximos estudios.

En lo que respecta a las limitaciones de este estudio, se hace necesario mencionar que sólo se analizaron las variables que la base de datos contenía y no fue posible estudiar algunas otras de interés de los autores, como por ejemplo describir la relación entre la afasia y la escolaridad o estudiar los subtipos de afasia, entre otros temas. Existen publicaciones que han advertido que los pacientes con afasias expresivas tienden a ser más jóvenes que aquellos con afasias receptivas, lo que también habría sido interesante de analizar en esta población. Pese a que los datos del estudio PISCIS fueron recogidos entre los años 2000 y 2002 y que por ende la información se refiere necesariamente a este período, actualmente no existe a nivel nacional otro estudio poblacional de ACV que haya estimado la incidencia de esta patología de manera prospectiva y de esta magnitud. La selección del lugar para llevar a cabo el estudio PISCIS (Iquique) resulta interesante, debido a su realidad geográfica, ubicada a cientos de kilómetros de la capital y con características de desierto y mar, lo que la vuelve una ciudad con población cautiva al momento del estudio.

La afasia es una patología que trasciende las esferas exclusivas del lenguaje; genera un quiebre de gran magnitud en la biografía de las personas y tiene un impacto severo en la calidad de vida de aquellos que la presentan. Si bien en los últimos años se ha podido ver un avance en materias de ayuda mediante la implementación de programas de habilitación y rehabilitación para este tipo de pacientes en Chile, aún falta mucho por avanzar. Se requiere mejorar en la prevención, controlando los factores de riesgos modificables y educando a la población.

Gracias a los avances tecnológicos y científicos, los pacientes con ACV han logrado mayor sobrevida. No obstante, es posible observar individuos con secuelas de todo ámbito (cognitivo, motor y/o lenguaje, etc). Se hace urgente poder contar con equipos de rehabilitación que actúen precozmente para abordar este tipo de patologías, apoyar al paciente y su familia, optimizar los resultados funcionales y evitar el aumento de déficit neurológico. 
Agradecimientos: A la Sra. Marinella Mazzei, docente del Magister de Salud Pública de la Universidad de Chile por sus correcciones y comentarios constantes.

\section{Referencias}

1. Ministerio de Salud (MINSAL). Plan de Acción Ataque Cerebrovascular. $2^{\circ}$ Edición. 2014.

2. Lavados P, Sacks C, Prina L, Escobar A, Tossi C, Araya $\mathrm{F}$ et al. Incidence, 30 -day case- fatality rate, and prognosis of stroke in Iquique: a 2- year community- based prospective study (PISCIS project).Lancet. 2005; 365: 2206-14.

3. El Banco Mundial. Esperanza de vida al nacer, total. Hallado en: http://datos.bancomundial.org/indicador/ SP.DYN.LE00.IN. (Acceso el 28 de enero de 2014).

4. Ministerio de Salud (MINSAL). Guía Clínica Auge. Accidente Cerebro Vascular Isquémico en personas de 15 años y más. 2013 (Acceso el 2 noviembre de 2013). Disponible en: web.misal.cl/AUGE-GUIAS-CLINICAS.

5. Nogales-Gaete, Donoso A. Verdugo R, editores. Enfermedades Cerebrovasculares Isquémicas: aterotrombótica, lacunar y cardioembólicas. Tratado de Neurología Clínica. Chile, Editorial Universitaria; 2005; 229-41.

6. Reyes A, Piedra L, Fafebre F. Ataque cerebrovascular isquémico, etiología y características clínicas: un estudio transversal. Revista Médica HJCA 2014; 6: 21-6.

7. Bamford P. Sandercock M. Dennis J. Burn C. Warlow $\mathrm{CP}$. Classification and natural history of clinically identifiable subtypes of cerebral infarction. Lancet 1991; 337: 1521-6.

8. Banks JL, Marotta CA. Outcomes validity and reliability of the modified Rankin scale: implications for stroke clinical trials: a literature review and synthesis. Stroke. 2007 Mar; 38(3): 1091-6.
9. Croquelois A, Bogousslavsky J, Stroke aphasia: 1.500 consecutive patients. Cerebrovasc Dis 2011; 31 (4): 3929.

10. Pedersen $\mathrm{P}$, Jorgensen $\mathrm{H}, \mathrm{Nakayama} \mathrm{H}$, Raaschou H, Olsen T. Aphasia in acute stroke: Incidence, determinants and recovery. Annals of Neurology 1995; 38 (4): 659-66.

11. Wade D, David R, Enderby P. Aphasia after stroke: natural history and associated deficits. J NeurolNeurosurg Psychiatry 1986; 49: 11-16.

12. Flowers H, Silver F, Fang J, Rochon E, Martino R. The incidence, co-ocurrence and predictor of dysphagia, dysarthria and aphasia after first-ever acute ischemic stroke. Journal of Communication Disorders. 20013; 238-248.

13. Reyes A, Piedra L, Fafebre F. Ataque cerebrovascular isquémico, etiología y características clínicas: un estudio transversal. Revista Médica HJCA 2014; 6: 21-6.

14. Engelter S, Gostynski M, Papa S, Frei M, Born C, Ajdacic-Gross V, et al. Epidemiology of Aphasia Attributable to First Ischemic Stroke: Incidence, Severity, Fluency, Etiology and Thrombolysis. Stroke 2006; 37: 1379-84.

15. El Hachioui H, Lingsma H. Van de Sandt-Koenderman M. Long- term prognosis of aphasia after stroke. J Neurol Neurosurg Psychiatry 2013; 310-5.

16. Hier D, Yoon W, Mohr J, Price T, Wolf P. Gender and Aphasia in the Stroke Data Bank. Brain and Language 1994; 155-67.

17. De Renzi E, Faglioni P, Ferrari P. The Influence of sex and age on the incidence and type of aphasia. Cortex. 1980; 16 (4): 627-30.

18. Laska A, Hellblom A, Murray V, Kahan T, Von Arbin M. Aphasia in acute stroke and relation to outcome. Journal of Internal Medicine 2001; (249): 413-22.

19. Tatemichi T, Desmond D, Stern Y, Paik M, Sano M, Bagiella E. Cognitive impairment after stroke: frecuency, patterns and relationship to functional patterns. J Neurol Neurosurg Psychaitry 1994; 57: 202-7. 